\title{
Article \\ Parametric Optimization of Electric Discharge Machining of Metal Matrix Composites Using Analytic Hierarchy Process
}

\author{
Sarabjeet Singh Sidhu ${ }^{1}$, Timur Rizovich Ablyaz ${ }^{2, *}$, Preetkanwal Singh Bains ${ }^{3}$, Karim Ravilevich Muratov ${ }^{2}$, \\ Evgeny Sergeevich Shlykov ${ }^{2}$ and Vladislav Vitalyevich Shiryaev ${ }^{2}$ \\ 1 Mechanical Engineering Department, Sardar Beant Singh State University (Formerly Known as Beant College \\ of Engineering and Technology), Gurdaspur 143521, India; sarabjeetsidhu@yahoo.com \\ 2 Perm National Research Polytechnic University, 614000 Perm, Russia; karimur_80@mail.ru (K.R.M.); \\ kruspert@mail.ru (E.S.S.); vlad2117@gmail.com (V.V.S.) \\ 3 Mechanical Engineering Department, IKG Punjab Technical University, Kapurthala 144603, India; \\ preetbains84@gmail.com \\ * Correspondence: lowrider11-13-11@mail.ru
}

check for

updates

Citation: Sidhu, S.S.; Ablyaz, T.R.; Bains, P.S.; Muratov, K.R.; Shlykov, E.S.; Shiryaev, V.V. Parametric Optimization of Electric Discharge Machining of Metal Matrix

Composites Using Analytic Hierarchy Process. Micromachines 2021, 12, 1289. https://doi.org/10.3390/mi12111289

Academic Editors: Francesco Modica and Irene Fassi

Received: 24 August 2021

Accepted: 19 October 2021

Published: 21 October 2021

Publisher's Note: MDPI stays neutral with regard to jurisdictional claims in published maps and institutional affiliations.

Copyright: (C) 2021 by the authors. Licensee MDPI, Basel, Switzerland. This article is an open access article distributed under the terms and conditions of the Creative Commons Attribution (CC BY) license (https:/ / creativecommons.org/licenses/by/ $4.0 /)$.

\begin{abstract}
The present study reports on the method used to obtain the reliable outcomes for different responses in electric discharge machining (EDM) of metal matrix composites (MMCs). The analytic hierarchy process (AHP), a multiple criteria decision-making technique, was used to achieve the target outcomes. The process parameters were varied to evaluate their effect on the material erosion rate $(M E R)$, surface roughness (SR), and residual stresses $(\sigma)$ following Taguchi's experimental design. The process parameters, such as the electrode material ( $\mathrm{Cu}, \mathrm{Gr}, \mathrm{Cu}-\mathrm{Gr})$, current, pulse duration, and dielectric medium, were selected for the analysis. The residual stresses induced due to the spark pulse temperature gradient between the electrode were of primary concern during machining. The optimum process parameters that affected the responses were selected using AHP to figure out the most suitable conditions for the machining of MMCs.
\end{abstract}

Keywords: analytical hierarchy process; residual stress; metal erosion rate; surface roughness

\section{Introduction}

Composite materials have superior properties, such as high strength; high modulus, low coefficient of thermal expansion; and resistance to fatigue, corrosion, and wear. Due to these prominent properties and their high strength-to-weight ratios, composites are extensively used in numerous advanced engineering applications. Composites with different reinforcements (such as fibers or particles) are being researched widely for their use in several applications, including manufacturing and biomedical industries. The composite materials, usually called metal matrix composites (MMCs), consist of a metal or alloy in the ductile phase to absorb and equally distribute the external load and develop a percolating network to position the reinforced fibers or particles. Alongside this, a brittle constituent, i.e., reinforcement, is embedded in the metal matrix [1-3]. The combined properties of these constituents in MMCs allow for high strength and fractural properties to be attained, as well as high temperature resistance, making them suitable for applications in the automobile and aviation industries, such as braking systems, piston rods, piston pins, and brake discs [4]. These composites are also used as thermal management solutions for high energy density miniature electronic components, such as microprocessor lids, flip-chip lids, and microwave housing, and can replace high-cost materials such as titanium-based alloy [5-7]. With the presence of a soft matrix phase and hard reinforced particles in MMCs, precise machining with conventional methods is challenging in terms of avoiding degradation of the material properties.

Such difficulties can be overcome by adopting newer machining methods that can achieve the desired geometry with minimum damage to the material properties [8]. One 
such method for machining MMCs is electric discharge machining (EDM), which operates by generating controlled electric sparks to machine composite materials with complex geometries and provides a better surface quality with high dimensional accuracy. In this process, the tool electrode produces its replica in the workpiece material, producing a series of discrete electrical sparks that are generated within the dielectric medium. One of the reasons for the tremendous popularity of this process is its ability to machine complex internal contours, even in hard-to-cut materials, with negligible cutting forces [2,8]; however, in this process, the rapid change in temperature gradient of the machined surface results in sub-surface defects such as cracks, spalling, porosity, residual stresses, and metallurgical transformation [9].

For the effective ED machining of MMCs, a higher value of discharge current and shorter pulse-on time is generally recommended. EDM was highlighted by several researchers in the literature as an effective non-traditional machining technique used for shaping and machining of difficult-to-machine materials such as Al-SiC metal matrix composite [10]. In a similar study, a $\mathrm{ZrB}_{2}-40 \% \mathrm{Cu}$ composite electrode was reported as an alternative electrode with a better material removal rate and tool wear rate than a conventionally used copper tool [11]; however, the diametric overcut and surface roughness were better with the copper tool than the composite tool electrodes [12].

The output responses, such as the material erosion rate (MER), tool wear rate (TWR), and surface roughness (SR), have been studied and widely reported for the EDM process $[2,8]$; however, one critical factor that significantly affects the machined component's life is the residual stresses induced while machining. These stresses are quantified using destructive and non-destructive routes [13-15]. Non-destructive testing (NDT) X-ray diffraction techniques have been successfully used to evaluate the residual stresses in the materials, and accordingly the effect of the process parameters [16].

Many optimization techniques have been used to analyze the effects of non-conventional machining process parameters on the output responses, such as the MER, TWR, SR, and residual stresses. For instance, grey relational analysis has been applied to optimize EDM process parameters on $\mathrm{Al}-10 \% \mathrm{SiC}$ composites [17].The multi-regression method was used to establish the relationships between the input and output parameters of the wire EDM process [18].The lexicographic goal programming method was used for optimization of EDM process parameters while machining MMC [19].

So far, the previous studies have mostly reported on the optimization of MER, TWR, and SR, but very few studies have focused on optimizing the process parameters in order to minimize residual stresses in MMCs. EDM has been widely used for metals and alloys, although its application on MMCs and analyses of the resulting residual stresses have been limited. As such, the present study aims to establish the best process parameter settings for $65 \mathrm{vol} \% \mathrm{SiC} / \mathrm{A} 356.2$ and hybrid $10 \mathrm{vol} \% \mathrm{SiC}-5 \mathrm{vol} \%$ quartz/Al. Three output responses, namely the $M E R, \mathrm{SR}$, and residual stresses, are optimized using the analytic hierarchy process (AHP). The AHP is a decision-aiding tool that involves specifying a goal, measuring the relative importance (priorities), and choosing the relevant criteria [20,21]. One of the advantages of this tool is that it merges both qualitative and quantitative factors. The tool was formulated to exhibit the way the decision-maker thinks and determines the options based on weighted values. The tangible (objective) and non-tangible (subjective) factors can be efficiently coordinated and can provide reliable findings utilizing simple calculations. The AHP is also validated in various other fields, such as for issues linked to the economy, the stock industry, aircraft manufacture, transportation, and in the construction industry [22].

The objectives of this study are as follows:

- Analyze the influence of the EDM process parameters on the $65 \mathrm{vol} \% \mathrm{SiC} / \mathrm{A} 356.2$ (sample I, procured from CPS System, Dallas, TX, USA) and 10vol\% SiC-5vol\% quartz/ $\mathrm{Al}$ composites (sample II, produced by a controlled environmental stir casting process); 
- $\quad$ Evaluate the outcomes, such as the MER (metal erosion rate), SR (surface roughness), and $\sigma$ (residual stresses), utilizing the L18 Taguchi experimental design and optimizing the process parameters using AHP.

\section{Material and Methods}

\subsection{Material}

Two different variants of particulate-reinforced MMCs were used in this study. The material (65vol\% SiC / A356.2 metal matrix composite) used in the study was procured in rectangular plates from CPS, Boston, MA, USA. The other specimen used in this experiment was a hybrid metal matrix composite with $10 \mathrm{vol} \% \mathrm{SiC}-5 \mathrm{vol} \%$ quartz in aluminum, which was prepared using the in-house stir-casting method. The material composition was quantified using optical emission spectroscopy (Make: Arun Technology PolySpek-M spectrometer) as $0.384 \% \mathrm{Zn}, 0.498 \% \mathrm{Cu}, 0.424 \% \mathrm{Fe}, 2.063 \% \mathrm{Si}$, and $0.354 \% \mathrm{~Pb}$, with the balance $\%$ as $\mathrm{Al}$.

\subsection{Method}

The experiments were conducted on an OSCARMAX (SD550 ZNC, Taiwan) diesinking EDM machine using a conventional polarity with the selected tool electrodes. The workpieces were machined in EDM oil as a dielectric fluid and with a suspended powder form of copper $(5 \mathrm{~g} / \mathrm{L})$ and graphite $(5 \mathrm{~g} / \mathrm{L})$ in the dielectric medium. To ensure the uniform mixing of the suspended powder, a stirrer pedal fixed at $1400 \mathrm{rpm}$ was used during machining. Three tool electrode materials, namely (i) electrolytic copper, (ii) finedgrained graphite (Poco-EDM 3), and (iii) a copper-graphite composite (50\% Cu, Grade 673) were used for the experimental study. The electrodes were machined to a cylindrical shape with a diameter of $18 \mathrm{~mm}$.

The responses such as the $M E R, S R$, and $\sigma$ were measured after each experimental trial. The $M E R$ was measured in terms of weight loss per unit time using a digital weighing machine (Chyo-MJ-300). The surface roughness was measured at three different directions of the machined surface using a Mitutoyo (SJ-400) surface roughness analyzer. The developed residual stresses while machining are quantified by $X$-ray diffraction technique using a PANalytical'sX'PertPro MPD (Almelo, The Netherlands) diffractometer. The diffractometer used in this study was a horizontal, fixed, laboratory-based system. Table 1 shows the brief experimental conditions for the PANalytical'sX'PertPro X-ray stress analysis.

Table 1. Residual stress measurement conditions.

\begin{tabular}{|c|c|}
\hline Factors & Conditions \\
\hline Characteristic X-ray & $\mathrm{Cu}-\mathrm{K} \alpha 1+2$ \\
\hline Measurement method & $\Omega$-Diffractometer method \\
\hline Diffraction plane, (hkl) & $(422)$ \\
\hline Tube voltage, KV & 45 \\
\hline Tube current, mA & 40 \\
\hline 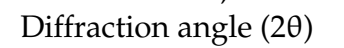 & $40^{\circ}-140^{\circ}$ \\
\hline Diffraction plane, (hkl) & $(422)$ \\
\hline
\end{tabular}

\subsection{Experimentation}

On the basis of the pilot study, workpiece material, dielectric type, tool electrode material, pulse-on and pulse-off durations, and current, the machining parameters were selected. The other factors, such as the open-circuit voltage ( 135 V) and flushing pressure $\left(0.6 \mathrm{~kg} / \mathrm{cm}^{2}\right)$, were maintained as constant during the experimental study. Table 2 shows the control factors and settings used for the experiments. 
Table 2. Parameters with different levels.

\begin{tabular}{cccc}
\hline \multirow{2}{*}{ Parameters (Symbol) } & \multicolumn{3}{c}{ Levels } \\
\cline { 2 - 4 } & Level-1 & Level-2 & Level-3 \\
\hline Workpiece $(\mathrm{WP})$ & $65 \mathrm{vol} \% \mathrm{SiC} / \mathrm{A} 356.2$ & 10 vol\% SiC-5 vol\% quartz/Al & - \\
Tool Electrode $(\mathrm{TE})$ & $(\mathrm{WP} \mathrm{I})$ & (WP II) & $\mathrm{Cu}-\mathrm{Gr}$ \\
Dielectric medium(D) & $\mathrm{Cu}$ & $\mathrm{Gr}$ & PMEDM \\
Current $(\mathrm{A}) \mathrm{Amp}$ & $\mathrm{EDM} \mathrm{oil} \mathrm{(D)}$ & PMEDM $(\mathrm{Cu})$ & $(\mathrm{Gr})$ \\
Pulse-on $\left(\mathrm{T}_{\text {on }}\right) \mu \mathrm{s}$ & 4 & 30 & 50 \\
Pulse-off $\left(\mathrm{T}_{\text {off }}\right) \mu \mathrm{s}$ & 10 & 30 & 45 \\
\hline
\end{tabular}

Because the chosen factors for the experiments involved a combination of two and three levels, the degree of freedom (dof) for2-level factors was 1 and the doffor3-level factors was 2; hence, the total dof required was 11 (i.e., 1 (one 2-level factor) $+5 \times 2$ (five 3-level factors) $=11$ ). The mixed-level orthogonal array involving a combination of two- and three-level factors with at least 11 dof was designated experimental design (DOE)L18. This DOE methodology used orthogonally designed arrays that significantly reduced the required number of experimental trials to record the necessary data without compromising the output data quality [23]. L18 signifies the18 distinct orthogonal trial conditions performed randomly to remove any undesirable inclinations in the study. The orthogonal arrays contained the two-level factor in column 1, with the option of assigning three-level factors to the other columns. The conditions of the experimental trials after the assignment of factors to a selected array are listed in Table 3. As can be seen from this design matrix, the first column represents the workpiece materials used in the study; thus, the first nine trials represent $65 \mathrm{vol} \% \mathrm{SiC} / \mathrm{A} 356.2 \mathrm{MMC}$, hereafter designated as WP I. The remaining nine trials (trials 10 to 18 ) represent $10 \mathrm{vol} \% \mathrm{SiC}-5 \mathrm{vol} \%$ quartz in aluminum, hereafter referred to as WP II. Other factors are assigned to the remaining columns of the L18 array, as listed in Table 3. The 18 experimental trials with two repetitions are performed as per Taguchi's design in random order. The mean MER, SR, and $\sigma$ are measured at the end of each trial and are presented in Table 3 under output responses. The MER is calculated by the weight difference of the workpiece before and after machining, as given by Equation (1):

$$
M E R=\frac{\left(w_{i}-w_{f}\right) 1000}{T} \mathrm{mg} / \mathrm{min}
$$

where $\Delta w$ if the change in weight, i.e., the weights before and after machining (gm); $\mathrm{t}$ is the machining time in minutes.

The SR was measured using a Mitutoyo SJ-400 surface roughness tester in terms of the arithmetic average of the absolute value Ra $(\mu \mathrm{m})$. Each sample was measured from three locations diametrically on the machined surface and was averaged for investigation.

Residual stresses were estimated with the help of the classical X-ray diffraction procedure. The peak diffracted from the (422) plane was selected to measure the shift at a maximum $2 \theta$ angle. The change in $\mathrm{d}$-spacing due to strain in the sample was minimal; hence, the highest possible $2 \theta$ angle peak was chosen. The relations of the $\mathrm{d}$-spacing $(\Delta \mathrm{d})$ with the diffraction peak $(\Delta \theta)$ is given by Equation (2):

$$
\frac{\Delta \mathrm{d}}{\Delta \theta}=(-)(\Delta \theta) \cot \theta
$$

The stresses were calculated using the classical $\sin ^{2} \psi$ technique $[14,16]$, with the assumption that the stress state is unidirectional. Equation (3) was used to calculate normal the residual stresses:

$$
\mathrm{a}^{+}=\frac{1}{2}\left(I e_{\varphi \psi+}+I e_{\varphi \psi-}\right)=\frac{1}{2} \mathrm{~S}_{2} \sin ^{2} \psi\left(\sigma_{\varphi}\right)+\operatorname{Ie} \varphi_{0}^{\circ}
$$


Here, parameter $\mathrm{a}^{+}$is the average of the lattice strain for positive $\left(\epsilon_{\varphi \psi+}\right)$ and negative $\left(\epsilon_{\varphi \psi-}\right)$ values and $\psi$ is the sample alignment (herein, $\left.\varphi=0^{\circ}\right) ; 1 / 2 \mathrm{~S}_{2}=(1+\nu) / \mathrm{E}, 1 / 2 \mathrm{~S}_{2}$ are the X-ray elastic constants (XEC's) and their values are represented in Table 4.

Table 3. Experimental layout (L18).

\begin{tabular}{|c|c|c|c|c|c|c|c|c|c|c|}
\hline \multirow{2}{*}{$\begin{array}{l}\text { Trial. No. } \\
\text { (T(n)) } \\
\text { Where } \\
\mathrm{n}=1-18\end{array}$} & \multicolumn{6}{|c|}{ Process Parameters } & \multicolumn{4}{|c|}{ Output Responses } \\
\hline & WP & TE & $\begin{array}{l}T_{\text {off }} \\
(\mu s)\end{array}$ & $\begin{array}{l}T_{\text {on }} \\
(\mu \mathrm{s})\end{array}$ & D & $\mathbf{A}$ & $\begin{array}{c}\sigma_{0} \\
(\mathrm{MPa})\end{array}$ & $\begin{array}{c}\sigma_{\mathrm{n}} \\
(\mathrm{MPa}) \text { Calculated from } \\
\text { Equation (5) }\end{array}$ & $\begin{array}{l}M E R \\
(\mathrm{mg} / \mathrm{min})\end{array}$ & $\begin{array}{c}\text { SR } \\
(\mu \mathrm{m})\end{array}$ \\
\hline 1 & WP I & 1 & 15 & 10 & 1 & 4 & 63.3 & 66.7 & 2.64 & 2.94 \\
\hline 2 & WP I & 1 & 30 & 30 & 2 & 8 & 74.6 & 55.4 & 14.275 & 2.05 \\
\hline 3 & WP I & 1 & 45 & 45 & 3 & 12 & 82.8 & 47.2 & 23.17 & 5.67 \\
\hline 4 & WP I & 2 & 15 & 45 & 2 & 8 & 36.3 & 93.7 & 23.38 & 2.09 \\
\hline 5 & WP I & 2 & 30 & 10 & 3 & 12 & 63.6 & 66.4 & 18.97 & 4.12 \\
\hline 6 & WP I & 2 & 45 & 30 & 1 & 4 & 110.3 & 19.7 & 3.04 & 3.00 \\
\hline 7 & WP I & 3 & 15 & 30 & 1 & 12 & 61.4 & 68.6 & 22.240 & 5.01 \\
\hline 8 & WP I & 3 & 30 & 45 & 2 & 4 & 78.5 & 51.5 & 9.860 & 2.06 \\
\hline 9 & WP I & 3 & 45 & 10 & 3 & 8 & 129 & 1 & 9.460 & 5.06 \\
\hline 10 & WP II & 1 & 15 & 30 & 3 & 8 & 70.4 & 162.1 & 20.90 & 6.69 \\
\hline 11 & WP II & 1 & 30 & 45 & 1 & 12 & 104 & 128.5 & 60.67 & 10.46 \\
\hline 12 & WP II & 1 & 45 & 10 & 2 & 4 & 78.1 & 154.4 & 10.860 & 4.69 \\
\hline 13 & WP II & 2 & 15 & 45 & 3 & 4 & 41.8 & 190.7 & 57.99 & 6.46 \\
\hline 14 & WP II & 2 & 30 & 10 & 1 & 8 & 149.3 & 83.2 & 18.86 & 8.44 \\
\hline 15 & WP II & 2 & 45 & 30 & 2 & 12 & 132.9 & 99.6 & 29.96 & 4.44 \\
\hline 16 & WP II & 3 & 15 & 10 & 2 & 12 & 77.7 & 154.8 & 65.5 & 6.76 \\
\hline 17 & WP II & 3 & 30 & 30 & 3 & 4 & 89.2 & 143.3 & 10.07 & 6.12 \\
\hline 18 & WP II & 3 & 45 & 45 & 1 & 8 & 231.5 & 1 & 45.72 & 7.95 \\
\hline
\end{tabular}

Table 4. X-ray elastic constants.

\begin{tabular}{lcc}
\hline & Sample I & Sample II \\
\hline Elastic constants $\mathbf{T}^{-\mathbf{1}} \mathbf{P a}\left(\mathbf{1} / \mathbf{2} \mathbf{S}_{\mathbf{2}}\right)$ & 6.98 & 16.84 \\
\hline
\end{tabular}

Equation (4) can be utilized to estimate the shear residual stress in further studies:

$$
\mathrm{a}^{-}=\frac{1}{2}\left(I e_{\varphi \psi+}-I e_{\varphi \psi-}\right)=\frac{1}{2} S_{2} \sin (2 \psi)\left(\tau_{\varphi}\right)
$$

The sample calibration for the stress test is represented below.

Sample calculation of $\sigma$ for trial 2 (WP I): The machined specimen was sectioned to $25 \times 25 \mathrm{~mm}$ using a wire-cut EDM machine. To limit modifications of the machined surface properties by heating in the wire EDM operation, we ensured that the cutting edge was far away from the calibration surface zone. The etching of re-solidified metal on the machined zone resulted in reduced computation errors. Residual stress was estimated in the aluminum matrix phase of the machined specimen. The measurement was accomplished by selecting the isolated peak diffracted at the highest value of $2 \theta$ from the plane. Figure 1 shows the X-ray spectra for trial 2. From the obtained X-ray spectra, the peak selected for residual stress determination was at approximately $137.23^{\circ}$.

Table 5 represents the various parameters for trial 2 used to measure residual stress at different $20 \psi$-tilts (positive and negative).

The normal residual stress was analyzed by comparing the linear fit regression equation obtained from the plot of a+vs. $\sin 2 \psi$ with Equation (3), i.e., the equation obtained was:

$$
\mathrm{a}^{+}=\left(5.21 * \sin ^{2} \psi=0.291\right) * 10^{-4} 5.21 \times 10^{-4}=\frac{1}{2} \mathrm{~S}_{2}\left(\sigma_{\varphi}\right)
$$

where $1 / 2 \mathrm{~S}_{2}=6.98 \mathrm{~T}^{-1} \mathrm{~Pa}, \varphi=0$

As such, the normal residual stress $\left(\sigma_{0}\right)$ for trial 2 was $74.6 \mathrm{MPa}$. 


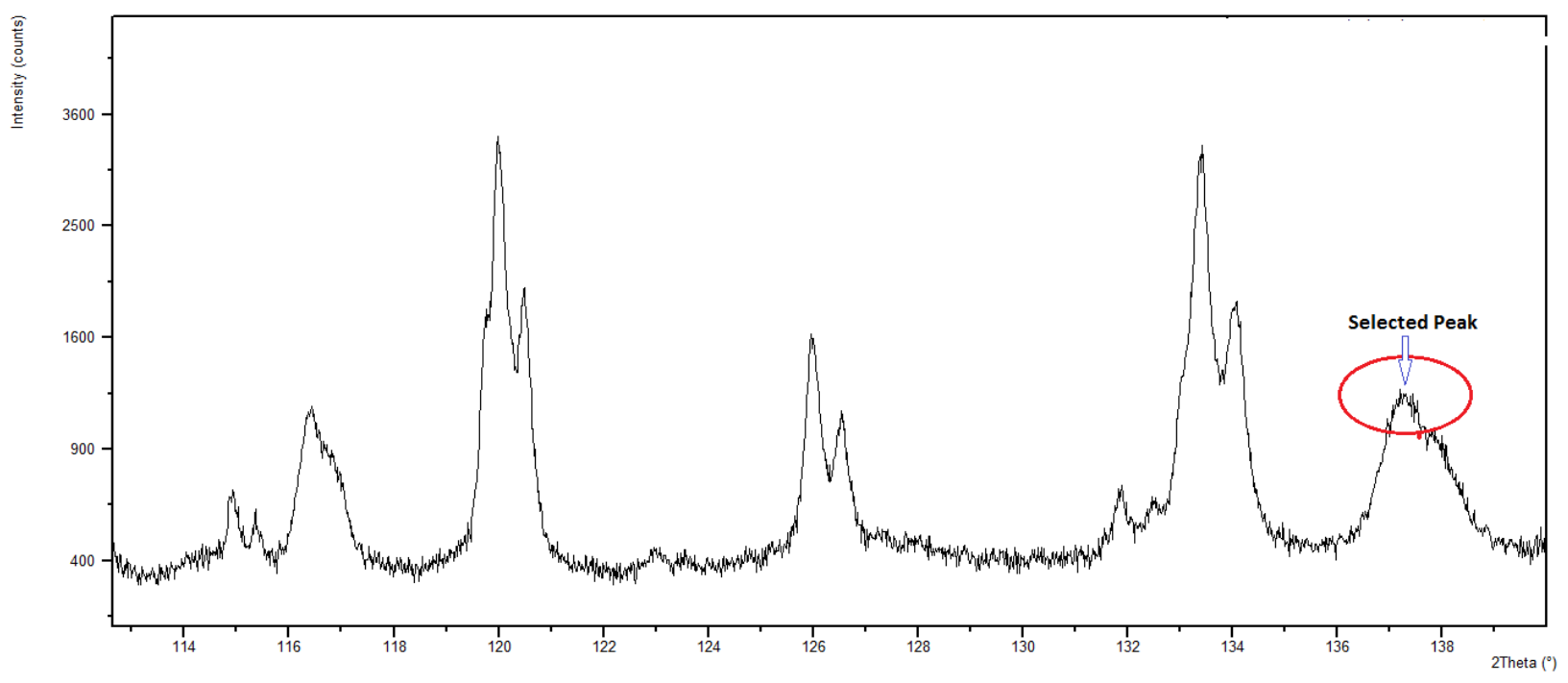

2 Theta (0)

Figure 1. Selected X-ray spectra peaks for residual stress calibration.

Table 5. X-ray spectra of lattice strain intrial 2.

\begin{tabular}{|c|c|c|c|c|c|c|}
\hline & $\psi$ & $\operatorname{Sin}^{2} \psi$ & $d_{\varphi \psi}$ & $\epsilon_{\psi \varphi}$ & $a+$ & $\mathbf{a}-$ \\
\hline & 0 & 0 & 0.827269 & 0 & & \\
\hline \multirow{10}{*}{ 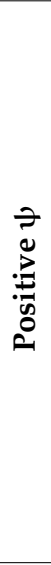 } & 12.92 & 0.05 & 0.827286 & 0.00002050 & $5.08 \times 10^{-5}$ & $-3.02 \times 10^{-5}$ \\
\hline & 18.44 & 0.1 & 0.827311 & 0.00005060 & $-1.70 \times 10^{-5}$ & $6.76 \times 10^{-5}$ \\
\hline & 22.79 & 0.15 & 0.827472 & 0.00024500 & $1.97 \times 10^{-4}$ & $4.82 \times 10^{-5}$ \\
\hline & 26.57 & 0.2 & 0.827425 & 0.00018900 & $2.48 \times 10^{-4}$ & $-5.90 \times 10^{-5}$ \\
\hline & 30.00 & 0.25 & 0.827450 & 0.00021900 & $1.84 \times 10^{-4}$ & $3.52 \times 10^{-5}$ \\
\hline & 33.21 & 0.3 & 0.827499 & 0.00027800 & $9.01 \times 10^{-5}$ & $1.88 \times 10^{-4}$ \\
\hline & 36.27 & 0.35 & 0.827564 & 0.00035700 & $2.10 \times 10^{-4}$ & $1.47 \times 10^{-4}$ \\
\hline & 39.23 & 0.4 & 0.827434 & 0.00020000 & $1.11 \times 10^{-4}$ & $8.91 \times 10^{-5}$ \\
\hline & 42.13 & 0.45 & 0.827673 & 0.00048800 & $2.94 \times 10^{-4}$ & $1.94 \times 10^{-4}$ \\
\hline & 45 & 0.5 & 0.827543 & 0.00033100 & $3.58 \times 10^{-4}$ & $-2.73 \times 10^{-5}$ \\
\hline \multirow{10}{*}{ 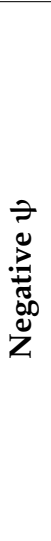 } & 12.92 & 0.05 & 0.827336 & 0.00008099 & \multirow{10}{*}{\multicolumn{2}{|c|}{$\begin{aligned} \mathrm{a}^{+}= & \frac{1}{2}\left(I_{\varphi \psi+}+I e_{\varphi \psi-}\right) \\
& (\text { fornormalstress }) \\
\mathrm{a}^{-}= & \frac{1}{2}\left(\text { I }_{\varphi \psi+}-I e_{\varphi \psi-}\right) \\
& (\text { forshearstress }) \\
& \text { where } \varphi=0\end{aligned}$}} \\
\hline & 18.44 & 0.1 & 0.827199 & -0.00008462 & & \\
\hline & 22.79 & 0.15 & 0.827392 & 0.00014868 & & \\
\hline & 26.57 & 0.2 & 0.827523 & 0.00030703 & & \\
\hline & 30.00 & 0.25 & 0.827392 & 0.00014868 & & \\
\hline & 33.21 & 0.3 & 0.827188 & -0.00009791 & & \\
\hline & 36.27 & 0.35 & 0.827321 & 0.00006286 & & \\
\hline & 39.23 & 0.4 & 0.827287 & 0.00002176 & & \\
\hline & 42.13 & 0.45 & 0.827351 & 0.00009912 & & \\
\hline & 45 & 0.5 & 0.827588 & 0.00038561 & & \\
\hline
\end{tabular}

2.4. Analysis of Variance for MER, SR, Residual Stress

The $M E R, \mathrm{SR}$, and $\sigma$ results were analyzed using analysis of variance (ANOVA). A summary of the ANOVA used for $M E R, S R$, and $\sigma$ is presented in Table 6 . The significant 
parameters were chosen by comparing the F-values with F-critical at a confidence level of $95 \%$. The higher the F-value, the greater the effect of the parameter on the responses.

Table 6. Analysis of variance for MER, SR, and $\sigma$.

\begin{tabular}{|c|c|c|c|c|c|c|c|c|c|c|}
\hline \multirow{2}{*}{ Factors } & \multirow{2}{*}{ dof } & \multicolumn{3}{|c|}{ Sum of Squares } & \multicolumn{3}{|c|}{ Variance } & \multicolumn{3}{|c|}{ F-Value } \\
\hline & & $M E R$ & SR & $\sigma$ & $M E R$ & SR & $\sigma$ & $M E R$ & SR & $\sigma$ \\
\hline $\mathrm{W} / \mathrm{Pc}$ & 1 & 1977.26 & 50.0333 & 4204.4 & 1977.26 & 50.0333 & 4204.4 & $10.50 *$ & $80.99 *$ & $9.13 *$ \\
\hline Electrode & 2 & 62.50 & 1.9590 & 3284.0 & 31.25 & 0.9795 & 1641.98 & 0.17 & 1.59 & $3.57 *$ \\
\hline Pulse-off & 2 & 435.50 & 0.9768 & 14262.5 & 217.75 & 0.4884 & 7131.27 & 1.16 & 0.79 & 15.49 * \\
\hline Pulse-on & 2 & 1448.53 & 4.6520 & 110.5 & 724.26 & 2.3260 & 55.26 & $3.85 *$ & $3.77 *$ & 0.12 \\
\hline $\begin{array}{l}\text { Dielectric } \\
\text { medium }\end{array}$ & 2 & 10.67 & 22.5037 & 6526.1 & 5.33 & 11.2519 & 3263.04 & 0.03 & $18.21 *$ & $7.09 *$ \\
\hline Current & 2 & 1494.86 & 10.6571 & 4725.5 & 747.43 & 5.3286 & 2362.75 & $3.97 *$ & $8.63 *$ & $5.13 *$ \\
\hline Error & 6 & 1129.73 & 3.7064 & 2762.6 & 188.29 & 0.6177 & 460.44 & & & \\
\hline Total & 17 & 6559.04 & 94.4886 & 35875.7 & & & & & & \\
\hline
\end{tabular}

* Significant factor.

MER: The ANOVA results show that the current and pulse-on contributed significantly to changes in MER. Additionally, the variations in the workpiece material had significant effects on the MER. On the contrary, the dielectric medium, pulse-off time, and electrode material had no significant effect. It was observed that with increases in pulse-on time and current, the $M E R$ increases significantly, since increases in the current and pulse-on time increase the spark energy duration; thus, with increased heat input, the temperature increases, resulting in the workpiece's higher melting or evaporation rate;

$S R$ : The surface roughness of the machined surface was significantly influenced by factors such as the powder concentration, current, and pulse-on time. Furthermore, the two materials showed quite different SR values. The roughness increased with increases in current and pulse-on time, whereas the powder in the dielectric medium improved the surface finish. An increase in current or pulse-on time increases the spark energy, which drives the formation of bigger and deeper craters, leading to a rough machined surface. The addition of powder consistently improved the finish of the machined surfaces; the spark becomes more uniform with increased frequency and widens the spark gap. This reduced the magnitude of the impact forces, resulting in small and shallow craters and lowering the surface roughness [24,25];

Residual Stress: The ANOVA results show that the pulse off time, powder mixing in the dielectric medium, and current significantly affected the $\sigma$. Additionally, the selected MMCs showed different residual stress values for similar parameter settings. It can be seen from the results that the pulse-on time affected the MER and SR but had no effect on $\sigma$. On the other hand, the pulse-off time hada significant impact on the development of residual stress due to the re-solidification time duration. The presence of suspended particles in the dielectric medium facilitates the easy formation of plasma channels between the electrode and the workpiece, resulting in lower SR and residual stress. The conductivity of suspended particles plays the major role in determining the SR but has no impact on the development of $\sigma$.

\section{Analytical Hierarchical Process}

Optimizing the responses independently results in vastly different parametric combinations of the machining process parameters. For example, if $M E R$ was optimized separately, this would cause some parameters to increase MER (the higher MER is, the better the function); however, these parameters may not result in decreased SR. The opposite would be true if SR was optimized individually. The identical condition involves residual stresses optimization. To obtain a result that is close to the target, the responses must be optimized together according to their priority. The analytical hierarchy process (AHP) offers one technique that suggested the best combination of parameters to reach or nearly reach the target. The AHP is simply structured and widely used for multiple-goal 
decision-making techniques and is classified as a decision-making tool for use under conditions of certainty, i.e., the data are obtained deterministically and the tool is designed for situations in which ideas, feelings, and emotions are quantified into a numerical scale [26]. The main steps used in the implementation of the AHP are as follows:

- Define the objective and evaluation criteria and develop the hierarchical structure, with an objective at the top level, the criteria and sub-criteria at the intermediate level, and the available alternatives at the lowest level;

- Form a pair-wise comparison matrix for each level with respect to the higher level and determine the relative importance of the different alternatives with respect to its immediately superior sub-criteria. The comparison is made on a 9-point "fundamental scale of Saaty", as represented in Table 7.

- Compute the relative weights for the pair-wise comparison matrices using eigenvector methods;

- Judge the scope of inconsistency by using the largest eigenvector. The judgment of the accepted degree of consistency can be checked by means of the consistency ratio $(\mathrm{CR})$ of the consistency index $(\mathrm{CI})$ with the appropriate value of the random index (RI) from Table 8.

- Repeat the above steps for all levels in the hierarchy, with the overall relative value evaluated by the linear addition function.

Table 7. Saaty's fundamental scale.

\begin{tabular}{cc}
\hline Scale Value & Explanation \\
\hline 1 & Equally preferred \\
3 & Slightly more preferred \\
5 & Strongly preferred \\
7 & Very strongly preferred \\
9 & Extremely preferred \\
$2,4,6,8$ & Used to reflect compromise between scale values \\
\hline
\end{tabular}

Table 8. Random consistency index.

\begin{tabular}{cccccccccccccc}
\hline $\boldsymbol{k}$ & $\mathbf{1}$ & $\mathbf{2}$ & $\mathbf{3}$ & $\mathbf{4}$ & $\mathbf{5}$ & $\mathbf{6}$ & $\mathbf{7}$ & $\mathbf{8}$ & $\mathbf{9}$ & $\mathbf{1 0}$ & $\mathbf{1 1}$ & $\mathbf{1 2}$ & $\mathbf{1 3}$ \\
\hline $\mathrm{RI}$ & 0.00 & 0.00 & 0.58 & 0.90 & 1.12 & 1.24 & 1.32 & 1.41 & 1.45 & 1.49 & 1.51 & 1.48 & 1.48 \\
\hline
\end{tabular}

The steps are summarized in Figure 2.

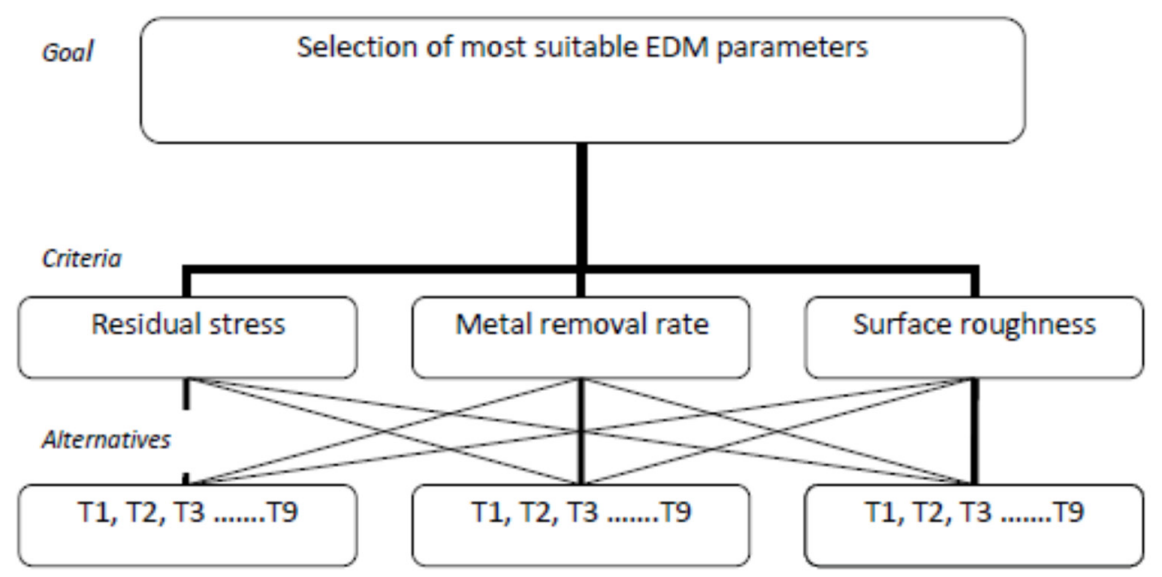

Figure 2. Hierarchy layout of the analytical hierarchy process.

The MMCs used in the present study are used for very high-end applications in aerospace and mining, while the residual stresses developed during the EDM process affect 
the service life of the product. Residual stress was assigned the maximum weight, followed by the material removal rate and surface roughness. To attain the desired objective, the residual stress results were slightly modified (cost-to-benefit conversion, which can be achieved by using the -ve sign), as follows:

$$
\sigma_{\mathrm{n}}=\left(\sigma_{\max }-\sigma_{0}\right)+1
$$

where $\sigma_{\max }$ is the maximum value of the residual stress in the corresponding trial set for each workpiece, $\sigma_{0}$ is the residual stress measured with the X-ray diffraction method, and $\sigma_{\mathrm{n}}$ is a modified residual stress value (refer to Table 3), which was calculated from Equation (5).

Using the criteria for assigning weights to the residual stress, $M E R$, and SR, a $(3 \times 1)$ weight column matrix, as shown in Table 9, was established for pair-wise comparison.

Table 9. AHP pair-wise comparison of weighting criteria.

\begin{tabular}{|c|c|c|c|c|}
\hline & $\sigma$ & $M E R$ & SR & Priority Vector \\
\hline$\sigma$ & 1 & 2 & 5 & 0.581552 \\
\hline$M E R$ & $1 / 2$ & 1 & 3 & 0.308996 \\
\hline \multirow[t]{2}{*}{ SR } & $1 / 5$ & $1 / 3$ & 1 & 0.109452 \\
\hline & $\lambda_{\max }=3.00369$ & $\mathrm{CI}=0.00184$ & CK & \\
\hline
\end{tabular}

Subsequently a pair-wise comparison of the experimental trials (alternatives) was developed for, $\sigma_{\mathrm{n}}, M E R$, and SR for each workpiece, with the results shown in Tables 10-15. The synthesized matrix to obtain priority vector of $\sigma$ for WP I is shown in Table 16. It was also ensured during pair-wise comparisons of alternatives that if the values attained during comparison were greater than the maximum limit of Saaty's fundamental scale, the highest value of the scale (9) was selected to avoid inconsistency.

Table 10. Pair-wise comparison of $\sigma$ values against their alternatives for WP I.

\begin{tabular}{|c|c|c|c|c|c|c|c|c|c|c|}
\hline . & T1 & T2 & T3 & $\mathbf{T} 4$ & T5 & T6 & T7 & T8 & T9 & Priority Vector \\
\hline T1 & 1 & 1 & 1 & 1 & 1 & 3 & 1 & 1 & 9 & 0.130829 \\
\hline T2 & 1 & 1 & 1 & $1 / 2$ & 1 & 3 & 1 & 1 & 9 & 0.12105 \\
\hline T3 & 1 & 1 & 1 & $1 / 2$ & 1 & 2 & 1 & 1 & 9 & 0.115577 \\
\hline T4 & 1 & 2 & 2 & 1 & 1 & 5 & 1 & 2 & 9 & 0.180525 \\
\hline T5 & 1 & 1 & 1 & 1 & 1 & 3 & 1 & 1 & 9 & 0.130829 \\
\hline T6 & $1 / 3$ & $1 / 3$ & $1 / 2$ & $1 / 5$ & $1 / 3$ & 1 & $1 / 4$ & $1 / 3$ & 9 & 0.050517 \\
\hline T7 & 1 & 1 & 2 & 1 & 1 & 4 & 1 & 1 & 9 & 0.136302 \\
\hline T8 & 1 & 1 & 1 & $1 / 2$ & 1 & 3 & 1 & 1 & 9 & 0.12105 \\
\hline \multirow[t]{2}{*}{ T9 } & $1 / 9$ & $1 / 9$ & $1 / 9$ & $1 / 9$ & $1 / 9$ & $1 / 9$ & $1 / 9$ & $1 / 9$ & 1 & 0.0133204 \\
\hline & & \multicolumn{3}{|c|}{$\lambda_{\max }=9.23034$} & & 0.0 & RI & $\mathrm{R}$ & & \\
\hline
\end{tabular}

Table 11. Pair-wise comparison of $M E R$ values with respect to their alternatives for WP I.

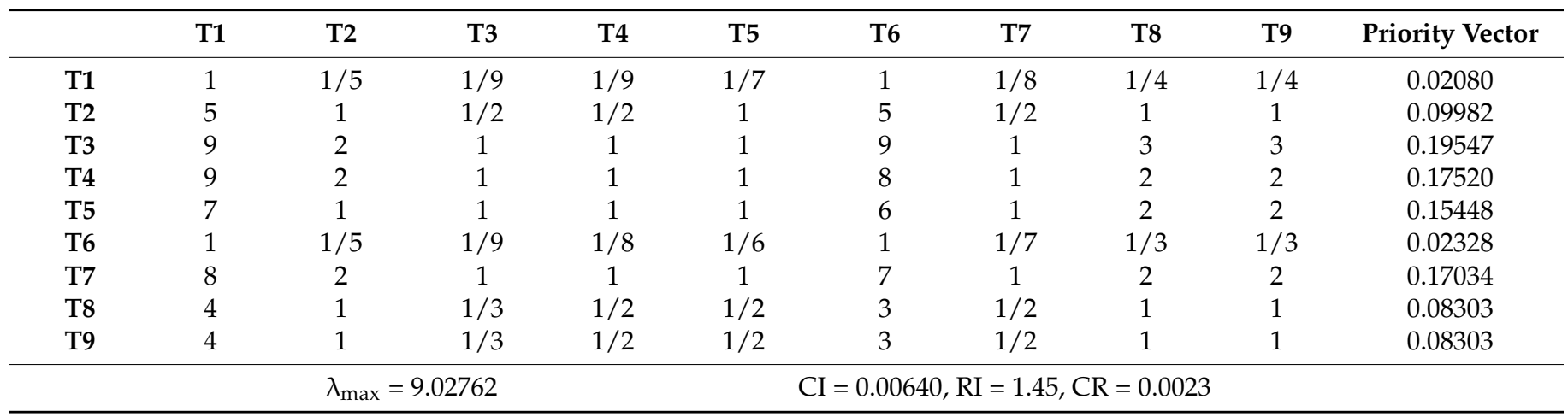


Table 12. Pair-wise comparison of SR values with respect to their alternatives for WP I.

\begin{tabular}{ccccccccccc}
\hline & T1 & T2 & T3 & T4 & T5 & T6 & T7 & T8 & T9 & Priority Vector \\
\hline T1 & 1 & 1 & $1 / 2$ & 1 & 1 & 1 & $1 / 2$ & 1 & $1 / 2$ & 0.0834519 \\
T2 & 1 & 1 & $1 / 3$ & 1 & $1 / 2$ & 1 & $1 / 2$ & 1 & $1 / 2$ & 0.0728733 \\
T3 & 2 & 3 & 1 & 3 & 1 & 2 & 1 & 3 & 1 & 0.176214 \\
T4 & 1 & 1 & $1 / 3$ & 1 & $1 / 2$ & 1 & $1 / 2$ & 1 & $1 / 2$ & 0.0728733 \\
T5 & 1 & 2 & 1 & 2 & 1 & 1 & 1 & 2 & 1 & 0.133865 \\
T6 & 1 & 1 & $1 / 2$ & 1 & 1 & 1 & $1 / 2$ & 1 & $1 / 2$ & 0.0834519 \\
T7 & 2 & 2 & 1 & 2 & 1 & 1 & 1 & 2 & 1 \\
T8 & 1 & 1 & $1 / 3$ & 1 & $1 / 2$ & $1 / 2$ & 1 & 1 & $1 / 2$ & 0.152199 \\
T9 & 2 & 2 & 1 & 2 & 1 & 1 & $1 / 2$ & 2 & 1 \\
\hline
\end{tabular}

Table 13. Pair-wise comparison of $\sigma$ values with respect to their alternatives for WP II.

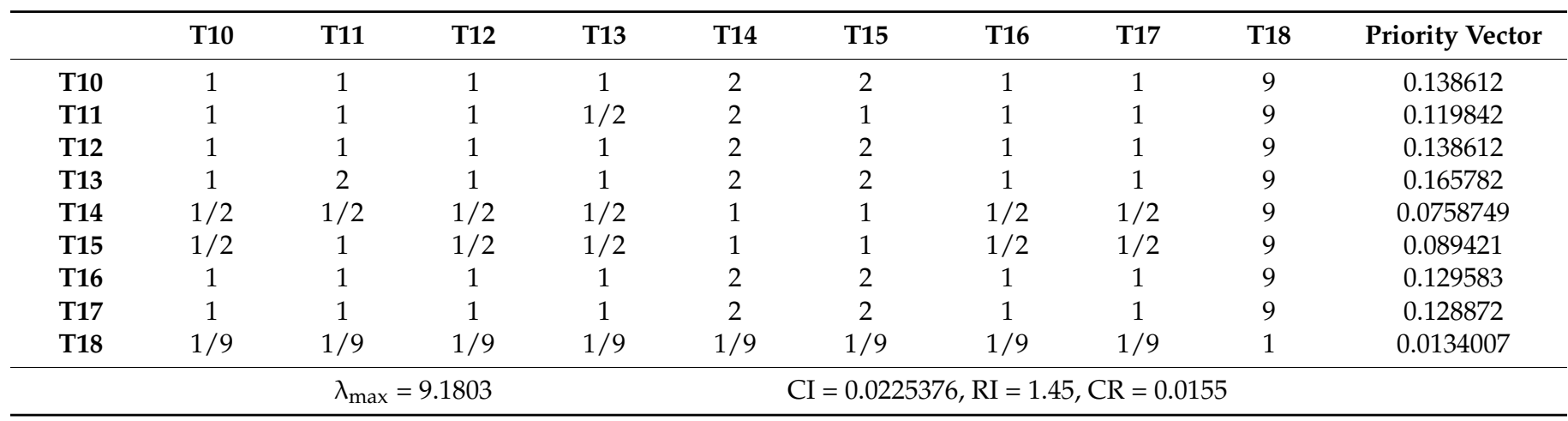

Table 14. Pair-wise comparison of $M E R$ values with respect to their alternatives for WP II.

\begin{tabular}{|c|c|c|c|c|c|c|c|c|c|c|}
\hline & T10 & T11 & T12 & T13 & T14 & T15 & T16 & T17 & T18 & Priority Vector \\
\hline T10 & 1 & $1 / 3$ & 2 & $1 / 3$ & 1 & 1 & $1 / 3$ & 2 & $1 / 2$ & 0.068095 \\
\hline T11 & 3 & 1 & 6 & 1 & 3 & 2 & 1 & 6 & 1 & 0.185247 \\
\hline T12 & $1 / 2$ & $1 / 2$ & 1 & $1 / 5$ & $1 / 2$ & $1 / 3$ & $1 / 6$ & 1 & $1 / 4$ & 0.0330161 \\
\hline T13 & 3 & 3 & 3 & 1 & 3 & 2 & 1 & 6 & 1 & 0.181600 \\
\hline T14 & 1 & 1 & 1 & 1 & 1 & $1 / 2$ & $1 / 3$ & 2 & $1 / 2$ & 0.0629946 \\
\hline T15 & 1 & 1 & 1 & 1 & 1 & 1 & $1 / 2$ & 3 & $1 / 2$ & 0.0923417 \\
\hline T16 & 3 & 3 & 3 & 3 & 3 & 3 & 1 & 6 & 1 & 0.1852470 \\
\hline T17 & $1 / 2$ & $1 / 2$ & $1 / 2$ & $1 / 2$ & $1 / 2$ & $1 / 2$ & $1 / 2$ & 1 & $1 / 5$ & 0.0314636 \\
\hline \multirow[t]{2}{*}{ T18 } & 2 & 2 & 2 & 2 & 2 & 2 & 2 & 2 & 1 & 0.159995 \\
\hline & & \multicolumn{3}{|c|}{$\lambda_{\max }=9.05235$} & \multicolumn{5}{|c|}{$\mathrm{CI}=0.00654334, \mathrm{RI}=1.45, \mathrm{CR}=0.00451$} & \\
\hline
\end{tabular}

Table 15. Pair-wise comparison of SR values with respect to their alternatives for WP II.

\begin{tabular}{ccccccccccc}
\hline & T10 & T11 & T12 & T13 & T14 & T15 & T16 & T17 & T18 & Priority Vector \\
\hline T10 & 1 & $1 / 2$ & 1 & 1 & 1 & 2 & 1 & 1 & 1 & 0.107181 \\
T11 & 2 & 1 & 2 & 2 & 1 & 2 & 2 & 2 & 1 & 0.171361 \\
T12 & 1 & $1 / 2$ & 1 & 1 & $1 / 2$ & 1 & 1 & 1 & $1 / 2$ & 0.0858196 \\
T13 & 1 & $1 / 2$ & 1 & 1 & 1 & 1 & 1 & 1 & 1 & 0.0990902 \\
T14 & 1 & 1 & 2 & 1 & 1 & 2 & 1 & 1 & 1 & 0.126548 \\
T15 & $1 / 2$ & $1 / 2$ & 1 & 1 & $1 / 2$ & 1 & $1 / 2$ & 1 & $1 / 2$ & 0.0741207 \\
T16 & 1 & $1 / 2$ & 1 & 1 & 1 & 2 & 1 & 1 & 1 & 0.107181 \\
T17 & 1 & $1 / 2$ & 1 & 1 & 1 & 1 & 1 & 1 & 1 & 0.0990902 \\
T18 & 1 & 1 & 2 & 1 & 1 & 2 & 2 & 1 & 1 \\
\hline
\end{tabular}


Table 16. Synthesized matrix of $\sigma$ for WP I.

\begin{tabular}{|c|c|c|c|c|c|c|c|c|c|}
\hline & T1 & T2 & T3 & $\mathrm{T} 4$ & T5 & T6 & T7 & T8 & T9 \\
\hline T1 & 0.13434 & 0.10405 & 0.10405 & 0.17208 & 0.13433 & 0.12442 & 0.13585 & 0.11843 & 0.12329 \\
\hline T2 & 0.13434 & 0.10405 & 0.10405 & 0.08604 & 0.13433 & 0.12442 & 0.13585 & 0.11843 & 0.12329 \\
\hline T3 & 0.13434 & 0.10405 & 0.10405 & 0.08604 & 0.13433 & 0.08295 & 0.13585 & 0.11843 & 0.12329 \\
\hline T4 & 0.13434 & 0.20809 & 0.20809 & 0.17208 & 0.13433 & 0.20737 & 0.13585 & 0.23685 & 0.12329 \\
\hline T5 & 0.13434 & 0.10405 & 0.10405 & 0.17208 & 0.13433 & 0.12442 & 0.13585 & 0.11843 & 0.12329 \\
\hline T6 & 0.04478 & 0.05202 & 0.05202 & 0.03442 & 0.04478 & 0.04147 & 0.03396 & 0.03948 & 0.12329 \\
\hline T7 & 0.13434 & 0.20809 & 0.20809 & 0.17208 & 0.13433 & 0.16590 & 0.13585 & 0.11843 & 0.12329 \\
\hline T8 & 0.13434 & 0.10405 & 0.10405 & 0.08604 & 0.13433 & 0.12442 & 0.13585 & 0.11843 & 0.12329 \\
\hline T9 & 0.01493 & 0.01156 & 0.01156 & 0.01912 & 0.01493 & 0.00461 & 0.01509 & 0.01316 & 0.01370 \\
\hline
\end{tabular}

The pair-wise comparison $(9 \times 9$ matrix $)$ of the alternatives was completed by comparing and rounding off the response ratio obtained in experimental trials. For example, if trial 1 gives a value of 16.5 and trial 2 gives 4.5, then the response ratio is the ratio of the values of the two trials (trial1/trial2), which is 3.66 (rounding off $=4$ ). The same procedure was adopted for all the response parameters to assign the weights. In the work [27] adopted percentage change in the state of tool wear while machining medium carbon steel workpiece. The change in percentage was used to assign the weight in pair-wise comparison matrix.

To illustrate this calculation, the pair-wise matrix for residual stress $(\sigma)$ (WP I) is considered, as shown in Table 16.

Step 1: The matrix was normalized by dividing each element of the matrix by its column total. For example, for the $\mathrm{T}(1,1)$ element, a value of 0.13434 was obtained by dividing 1 by the column total of $7.444(1+1+1+1+1+1 / 3+1+1+1 / 9)$. The same procedure was adopted for each element of the matrix, with the results given in Table 16.

Step 2: The estimation of the priority vector was done by taking the row average, i.e., $(0.13434+0.10405+0.10405+0.17208+0.13433+0.12442+0.13585+0.11843+0.12329)$ and dividing it by 9 .

Similarly, the synthesized pair-wise comparison matrix, priority vectors, and validation of the constructed matrix were performed for each response parameter. The overall weight was calculated by multiplying the alternative available priority vectors for each sample with the criteria weight, as given in Table 17.

Table 17. Overall weight matrix of WP I for the priority vector.

\begin{tabular}{cccccc}
\hline Trials & $\begin{array}{c}\boldsymbol{\sigma} \\
\mathbf{( 0 . 5 8 1 5 5 2 )}\end{array}$ & $\begin{array}{c}\text { MER } \\
\mathbf{( 0 . 3 0 8 9 9 6 )}\end{array}$ & $\begin{array}{c}\text { SR } \\
\mathbf{( 0 . 1 0 9 4 5 2 )}\end{array}$ & Overall Priority Vector & Ideal Weight Vector \\
\hline T1 & 0.130829 & 0.02080 & 0.0834519 & 0.091645 & 0.548453 \\
T2 & 0.12105 & 0.09982 & 0.0728733 & 0.109219 & 0.653623 \\
T3 & 0.115577 & 0.19547 & 0.176214 & 0.146899 & 0.879124 \\
T4 & $\mathbf{0 . 1 8 0 5 2 5}$ & $\mathbf{0 . 1 7 5 2 0}$ & $\mathbf{0 . 0 7 2 8 7 3 3}$ & $\mathbf{0 . 1 6 7 0 9 7}$ & $\mathbf{1 . 0 0 0 0 0 0 *}$ \\
T5 & 0.130829 & 0.15448 & 0.133865 & 0.138471 & 0.828682 \\
T6 & 0.050517 & 0.02328 & 0.0834519 & 0.045707 & 0.273534 \\
T7 & 0.136302 & 0.17034 & 0.152199 & 0.148560 & $0.889066^{* *}$ \\
T8 & 0.12105 & 0.08303 & 0.0728733 & 0.103185 & 0.617514 \\
T9 & 0.0133204 & 0.08303 & 0.152199 & 0.049217 & 0.294540 \\
\hline
\end{tabular}

$* 1$ st rank, ${ }^{* *} 2$ nd rank.

The overall priority for each EDM parameter setting was calculated as demonstrated below:

Overall weight of T1 (WP I): Overall Weight $=0.581552(0.130829)+0.308996(0.02080)+$ $0.1094520 .0834519)=0.091645$.

Similarly, the overall weight was calculated for each trial conducted for the selected workpieces. 
The remaining calculations were completed by combining the assigned criteria weight with the alternative priority weight to obtain the overall priority results (Tables 17 and 18), as per the hierarchical steps given in Figure 1.

Table 18. Overall weight matrix of WPII for the priority vector.

\begin{tabular}{cccccc}
\hline Trials & $\begin{array}{c}\boldsymbol{\sigma} \\
\mathbf{( 0 . 5 8 1 5 5 2 )}\end{array}$ & $\begin{array}{c}\text { MER } \\
\mathbf{( 0 . 3 0 8 9 9 6 )}\end{array}$ & $\begin{array}{c}\text { SR } \\
\mathbf{( 0 . 1 0 9 4 5 2 )}\end{array}$ & Overall Priority Vector & Ideal Weight Vector \\
\hline T10 & 0.138612 & 0.068095 & 0.107181 & 0.113791 & 0.73091 \\
T11 & 0.119842 & 0.185247 & 0.171361 & 0.146440 & 0.940701 \\
T12 & 0.138612 & 0.0330161 & 0.0858196 & 0.100555 & 0.645949 \\
T13 & $\mathbf{0 . 1 6 5 7 8 2}$ & $\mathbf{0 . 1 8 1 6 0 0}$ & $\mathbf{0 . 0 9 9 0 9 0 2}$ & $\mathbf{0 . 1 5 5 6 7 2}$ & $\mathbf{1 . 0 0 0 0 0 0 *}$ \\
T14 & 0.0758749 & 0.0629946 & 0.126548 & 0.077638 & 0.498728 \\
T15 & 0.089421 & 0.0923417 & 0.0741207 & 0.088929 & 0.571260 \\
T16 & 0.129583 & 0.1852470 & 0.107181 & 0.149991 & $0.963508 * *$ \\
T17 & 0.128872 & 0.0314636 & 0.0990902 & 0.095874 & 0.615871 \\
T18 & 0.0134007 & 0.159995 & 0.12661 & 0.071110 & 0.456796 \\
\hline
\end{tabular}

* 1 st rank, ${ }^{* *}$ 2nd rank.

The ideal weight vector was obtained by dividing the priority vector with the largest priority weight element in the matrix. The advantage of using an idealized weight vector is that the ranking of trials does not change due to the influence of a newly introduced non-optimal identical alternative [27].

From the calculated overall priority, the trials were ranked for each type of MMC. The maximum overall weight or composite performance score for sample I was obtained for T4 as given in Table 17 (See also Table 3), which was conducted with a graphite electrode; dielectric medium mixed with $\mathrm{Cu}$ powder; pulse-off and pulse-on times of $15 \mu \mathrm{s}$ and $45 \mu \mathrm{s}$, respectively; and current of 8 amps. Similarly, the maximum overall weight or composite performance score for sample II as obtained in Table 18 corresponds to trial number 13 (Table 3) of the original L18 array. This trial was also completed with a graphite electrode; dielectric medium mixed with graphite powder; pulse-off and pulse-on times of $15 \mu$ s and $45 \mu \mathrm{s}$, respectively; and a current setting of 4 amps. It was observed that the presence of powder in the dielectric medium expanded the area of the spark zone between the electrodes, thereby minimizing the impact of thermal shocks on the machined surface and diminishing the induced residual stresses; thus, the solution that globally optimizes residual stresses, MER, and SR for the two types of MMCs (WP I and WP II) used in the experiment was obtained (Table 19).

Table 19. Summarized process parameters for the target responses.

\begin{tabular}{ccc}
\hline Parameter & WP I & WP II \\
\hline Tool Electrode & Graphite & Graphite \\
Dielectric medium & PMEDM $(\mathrm{Cu})$ & PMEDM $(\mathrm{Gr})$ \\
Pulse-off time & $15 \mu \mathrm{s}$ & $15 \mu \mathrm{s}$ \\
Pulse-on time & $45 \mu \mathrm{s}$ & $45 \mu \mathrm{s}$ \\
Current & $8 \mathrm{Amp}$ & $4 \mathrm{Amp}$ \\
\hline
\end{tabular}

\section{Conclusions}

In the present study, three output response parameters, namely $\sigma, M E R$, and $\mathrm{SR}$, were optimized using a manageable AHP technique. Due to conflicting parameter settings for different output responses in the EDM process, identifying process parameters is a complex decision-making process. A manageable AHP approach was used in the present study to obtain a more reliable global composite performance score for various trial conditions in powder-mixed electric discharge machining (PMEDM) of MMCs. The process conditions that affected the three responses, namely $\sigma, M E R$, and SR, were identified and optimized using AHP for two different types of MMCs. The current and pulse-on time significantly affected $M E R$, while the addition of the powder, current, and pulse-on time influenced 
SR. Despite this, the pulse-off duration had no significant effect on MER or SR. Still, the pulse-off duration had the most considerable influence on residual stresses, followed by the dielectric medium, current, and type of tool electrode.

The three responses were optimized together according to the predetermined goal using AHP. The optimal process conditions for the selected materials were identified. The overall process for both workpieces revealed that machining the workpiece with a graphite tool electrode and higher pulse-on time setting coupled with lowest pulse-off time in the presence of a suspended particle dielectric medium (PMEDM) contributed to minimizing the residual stress with the desired MER. Due to the denser ceramic-reinforced particles in WP I compared to WP II, the target results were achieved at a higher current level (i.e., 8A) than the current required for WP II. The optimal settings for achieving the specified target results involved the graphite tool electrode coupled with pulse-on and -off times of $45 \mu \mathrm{s}$ and $15 \mu$ s, respectively, for both workpieces. The methodology used to obtain optimum EDM process parameters can be extended by prioritizing different responses (i.e., $M E R$, SR) according to the end-use application of the product. Overall, the use of AHP will open the horizon for EDM practitioners to determinevarious process parameters, improving their ability to achieve their desired targets.

Author Contributions: Conceptualization, S.S.S., P.S.B., E.S.S. and T.R.A.; methodology, S.S.S., K.R.M. and T.R.A.; validation, S.S.S. and T.R.A.; formal analysis, S.S.S., T.RA. and E.S.S.; investigation, S.S.S., E.S.S. and T.R.A.; resources, S.S.S., E.S.S., T.R.A., and K.R.M.; data curation, E.S.S., K.R.M. and V.V.S.; software, V.V.S.; writing—original draft preparation, S.S.S., P.S.B., E.S.S. and T.R.A.; writing—review and editing, S.S.S., K.R.M., T.R.A. and V.V.S.; visualization, S.S.S., E.S.S. and T.R.A.; supervision, S.S.S. and T.R.A.; project administration, S.S.S., K.R.M. and T.R.A. funding acquisition, T.R.A., E.S.S. and K.R.M. All authors have read and agreed to the published version of the manuscript.

Funding: Financial support was provided by the Russian Ministry of Science and Higher Education (project FSNM-2020-0028).

Conflicts of Interest: The authors declare no conflict of interest. The funders had no role in the design of the study; in the collection, analyses, or interpretation of data; in the writing of the manuscript, or in the decision to publish the results.

\section{References}

1. Callister, W.D. Composite. In Material Science and Engineering Material, 6th ed.; Wiley: New York, NY, USA, 2004 ; pp. 527-559.

2. Bains, P.S.; Sidhu, S.; Payal, H.S. Fabrication and Machining of Metal Matrix Composites: A Review. Mater. Manuf. Process. 2016, 31, 553-573. [CrossRef]

3. Ablyaz, T.; Bains, P.; Sidhu, S.; Muratov, K.; Shlykov, E. Impact of Magnetic Field Environment on the EDM Performance of Al-SiC Metal Matrix Composite. Micromachines 2021, 12, 469. [CrossRef]

4. Ahamed, A.R.; Asokan, P.; Aravindan, S. EDM of hybrid Al-SiCp-B4Cp and Al-SiCp-Glassp MMCs. Int. J. Adv. Manuf. Technol. 2009, 44, 520-528. [CrossRef]

5. Sidhu, S.S.; Kumar, S.; Batish, A. Metal Matrix Composites for Thermal Management: A Review. Crit. Rev. Solid State Mater. Sci. 2015, 41, 132-157. [CrossRef]

6. Dufour, B.; McNulty, M.; Miller, S. Microwave multi-chip module utilizing aluminum silicon carbide with in-situ cast components and high density interconnect technology. Int. J. Microcircuits Electron. Packag. 1997, 20, 303-308.

7. Novich, B.E.; Adams, R.W.; Occhionero, M.A. Low-Cost MCM-D Cavity Substrates for Packaging High Density Si and GaAs Devices. In Proceedings of the 10th European Microelectronics Conference, ISHM, Copenhagen, Denmark, 14-17 May 1995; pp. 475-481.

8. Sidhu, S.S.; Batish, A.; Kumar, S. Fabrication and electrical discharge machining of metal-matrix composites: A review. J. Reinf. Plast. Compos. 2013, 32, 1310-1320. [CrossRef]

9. Sidhu, S.; Batish, A.; Kumar, S. Study of Surface Properties in Particulate-Reinforced Metal Matrix Composites (MMCs) Using Powder-Mixed Electrical Discharge Machining (EDM). Mater. Manuf. Process. 2014, 29, 46-52. [CrossRef]

10. Bains, P.S.; Singh, S.; Sidhu, S.S.; Kaur, S.; Ablyaz, T.R. Investigation of Surface Properties of Al-SiC Composites in Hybrid Electrical Discharge Machining. In Structural Materials; Springer: Cham, Switzerland, 2018; pp. 181-196.

11. Rafaqat, M.; Mufti, N.A.; Ahmed, N.; AlAhmari, A.M.; Hussain, A. EDM of D2 Steel: Performance Comparison of EDM Die Sinking Electrode Designs. Appl. Sci. 2020, 10, 7411. [CrossRef]

12. Khanra, A.; Sarkar, B.; Bhattacharya, B.; Pathak, L.; Godkhindi, M. Performance of ZrB2-Cu composite as an EDM electrode. J. Mater. Process. Technol. 2007, 183, 122-126. [CrossRef] 
13. Withers, P.; Bhadeshia, H.K.D.H. Residual stress. Part 1-Measurement techniques. Mater. Sci. Technol. 2001, 17, 355-365. [CrossRef]

14. Welzel, U.; Ligot, J.; Lamparter, P.; Vermeulen, A.C.; Mittemeijer, E.J. Stress analysis of polycrystalline thin films and surface regions by X-ray diffraction. J. Appl. Crystallogr. 2005, 38, 1-29. [CrossRef]

15. Cuullity, B.D. Elements of X-ray Diffraction, 2nd ed.; Addison-Wesley: New York, NY, USA, 1978.

16. Sidhu, S.; Batish, A.; Kumar, S. Neural network-based modeling to predict residual stresses during electric discharge machining of Al/SiC metal matrix composites. Proc. Inst. Mech. Eng. Part B J. Eng. Manuf. 2013, 227, 1679-1692. [CrossRef]

17. Singh, P.; Raghukandan, K.; Rathinasabapathi, M.; Pai, B. Electric discharge machining of Al-10\%SiCP as-cast metal matrix composites. J. Mater. Process. Technol. 2004, 155-156, 1653-1657. [CrossRef]

18. Tzeng, Y.-F.; Chen, F.-C. Multi-objective optimisation of high-speed electrical discharge machining process using a Taguchi fuzzy-based approach. Mater. Des. 2007, 28, 1159-1168. [CrossRef]

19. Sidhu, S.S.; Batish, A.; Kumar, S. EDM of Metal Matrix Composite for Parameter Design Using Lexicographic Goal Programming. Mater. Manuf. Process. 2013, 28, 495-500. [CrossRef]

20. Saaty, T.L. A scaling method for priorities in hierarchical structures. J. Math. Psychol. 1977, 15, 234-281. [CrossRef]

21. Al-Harbi, K.M.-S. Application of the AHP in project management. Int. J. Proj. Manag. 2001, 19, 19-27. [CrossRef]

22. Whitaker, R. Validation examples of the Analytic Hierarchy Process and Analytic Network Process. Math. Comput. Model. 2007, 46, 840-859. [CrossRef]

23. Banerjee, S.; Sutradhar, G.; Sahoo, P. Design of experiment analysis of elevated temperature wear of Mg-WC nano-composites. Rep. Mech. Eng. 2021, 2, 202-211. [CrossRef]

24. Singh, G.; Ablyaz, T.R.; Shlykov, E.S.; Muratov, K.R.; Bhui, A.S.; Sidhu, S.S. Enhancing Corrosion and Wear Resistance of Ti6Al4V Alloy Using CNTs Mixed Electro-Discharge Process. Micromachines 2020, 11, 850. [CrossRef]

25. Haque, R.; Sekh, M.; Kibria, G.; Haidar, S. Improvement of Surface Quality of Ti-6Al-4VAlloy by Powder Mixed Electrical Discharge Machining Using Copper Powder. Facta Univ. Ser. Mech. Eng. 2021. [CrossRef]

26. Taha, H.A. Operation research an introduction. In Decision Analysis and Games, 7th ed.; Pearson Education: New York, NY, USA, 2002; pp. 503-511.

27. Das, S.; Chattopadhyay, A. Application of the analytic hierarchy process for estimating the state of tool wear. Int. J. Mach. Tools Manuf. 2003, 43, 1-6. [CrossRef] 